\title{
Modeling and Simulation on Errors of Feed Unit by Considering Change of Force Bearing Point
}

\author{
Wei Zhang ${ }^{1,2, a}$, Min Wang ${ }^{1}$ and Bing-bing Le ${ }^{1}$ \\ ${ }^{1}$ Advanced Manufacturing technology of the Key Laboratory of Beijing Municipality College of Mechanical \\ Engineering and Applied Electronics Technology, Beijing University of Technology, Beijing 100124, China \\ ${ }^{2}$ Engineering Training Center, Inner Mongolia University of Science and Technology, Baotou 014010, China
}

\begin{abstract}
The linear feed unit is a type of precision linear motion component that is widely used in computer numerical control (CNC) machine tools. The contact stiffness and error influences the performance of the feed unit directly. Thus, investigating contact stiffness and error is important in optimizing the design and improving the performance of the linear feed unit. In this study, the contact mechanics and the deformation of the roller between the roller and rail are analyzed. Calculation model of the contact stiffness and error based on the Hertz theory and multi-body kinematics are established and the change of the contact angle is also considered. Errors and the contact stiffness curve of five directions and the changes of the slope of the stiffness curve after the load increases to a certain size are obtained. The Motion precision errors of The roller linear feed unit are analyzed. The effectiveness of the proposed models on contact stiffness and error is verified through simulation on a specialized test system of the linear feed unit.
\end{abstract}

\section{Introduction}

The roller linear feed unit has widely used as a guide and load-bearing member in machine tools and mechanical devices. The feed unit has high positioning accuracy, low coefficient of friction, good precision retaining ability and good maintainability among others; thus, roller linear feed unit is a key feature of $\mathrm{CNC}$ machining equipment. The roller linear feed unit is mostly used for small or midsized precision machine tools, whereas the roller linear feed nnit has higher load capacity and higher

\footnotetext{
a Corresponding author: zhangwei2009035@163.com
} 
rigidity and is applicable to large $\mathrm{CNC}$ machine tools and machining centers. In recent years, the growing trend of high speed, high precision, and high durability in CNC machine tools has propelled high performance and reliability to be the most important index for linear feed unit. Thus, in-depth researches on the dynamic characteristics of linear feed unit are greatly needed. This research can lay a good foundation for enhancing the performance of linear feed unit.

Existing researches on the dynamic characteristics of linear feed unit were focused on contact stiffness, vibration during operation, and friction characteristics of roller linear guides. $\mathrm{Wu}[1,2]$ and Liu [3] studied the contact stiffness between the roller and raceway and its related influence factors were analyzed and calculated based on the Hertz theory. In addition, vibration synthesis and prediction by the method of employing the finite element were performed. After Shimizu's study [4,5] on the characterization of frictional hysteresis in roller-bearing guide ways, In article Jui Pin Hung[6], the linear rolling guide under different loading finite element model was established based on Hertz contact theory, the influence of external load to guide stiffness was studied and the finite element model was modified based on test results. In Wei Jun T [7], the relationship of the force between roller and raceway was analyzed and the stiffness model of horizontal, vertical and deflection angle around the spindle rotational was built based on Palmgren formula. The hertz contact model of roller among nails of linear roller guide is established firstly. Then the coordinate transformation matrix between the two coordinate systems is obtained with multi-body kinematic theory. Due to the analysis the stress of the roller linear motion unit, five mechanical equilibrium equations are obtained, then the amount of deformation of its five directions is determined. Thereafter, the analysis of roller linear feed units is simulated based on contact stiffness model proposed and the motion precision errors of the roller linear feed unit feed unit are analyzed. Finally, the proposed models are simulated by computer.

\section{Contact stiffness of linear feed unit}

The roller linear feed unit (hereinafter referred to as the table) contains four sliders; each slider also contains a number of rollers. The feed unit produces three rotation errors and two straightness errors with the role of the force of three directions, which are represented respectively as: $\delta y, \delta z, \varepsilon x, \varepsilon y, \varepsilon z$. $\delta y, \delta z$ are the deformation along $Y$ and $Z$ axis respectively, and $\varepsilon x, \varepsilon y, \varepsilon z$ are the deflection angle around the $X, Y, Z$ axis respectively. The coordinate system $O_{0}$ in the center of the working table is set up. The three axes are parallel to the movement direction, horizontal direction and vertical direction of the work table, and the center of the table and the slider are high in the vertical direction. The coordinate system $O_{1}(0, \delta \mathrm{y}, \delta \mathrm{z})$ is established in the center of the working table after the deformation, as shown in Figure. 1, In Figure 2, the black graphic slider with external loading produces linear roller guide way side position coordinates; the red graphic slider with external loading generates the position coordinates of elastic deformation. 

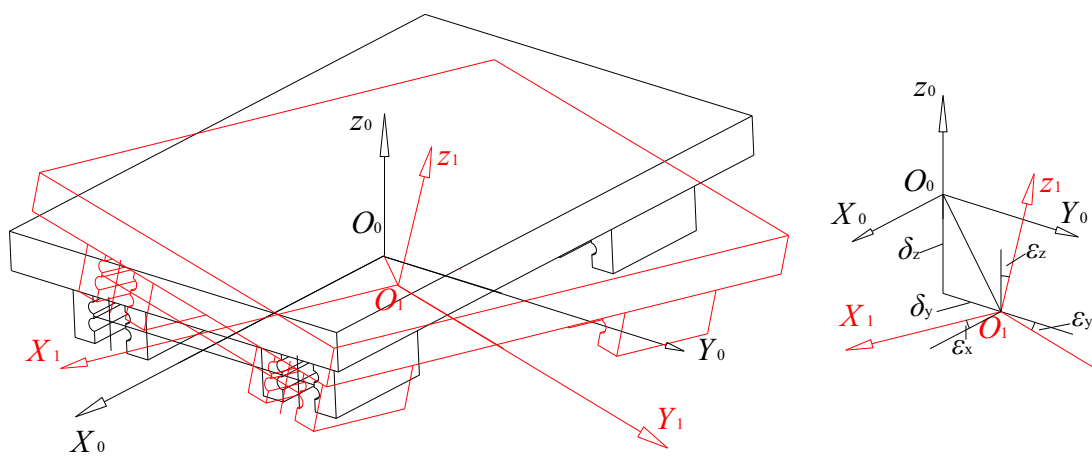

Figure.1 Coordinate system of Linear feed unit

The transformation matrix ${ }^{0} \boldsymbol{T}_{1}$ from the coordinate system of $O_{0}$ to the coordinate system of $O_{1}$ is:

$$
{ }^{0} T_{1}=\left[\begin{array}{cccc}
1 & -\varepsilon_{z} & \varepsilon_{y} & 0 \\
\varepsilon_{z} & 1 & -\varepsilon_{x} & \delta_{y} \\
-\varepsilon_{y} & \varepsilon_{x} & 1 & \delta_{z} \\
0 & 0 & 0 & 1
\end{array}\right]
$$

In the rolling linear guide, the roller, the slider and the track have contact surface. When the slider is subjected to external load, the contact stress and the elastic deformation are generated in the contact area at the same time. Under the condition of applying the pre pressure, the cross section of the slider 1 is analyzed. In Figure 2, the position of each column roller in slider and force are showed below.
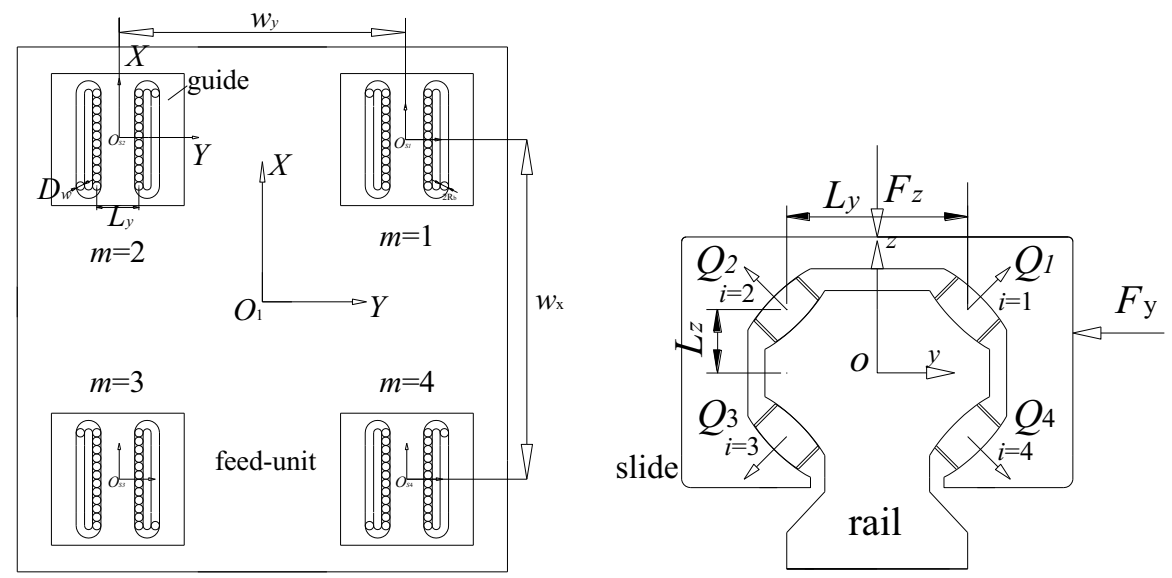

Figure.2 Locations and force of every roller in feed unit

As is shown in Figure.3, when there is no external load, according the geometry relationship of roller and rail the distance between the curvature center of the slider raceway and the guide raceway, is obtained $\boldsymbol{O}_{\mathbf{s}} \boldsymbol{O}_{\mathrm{g}}$ : equation (2) 


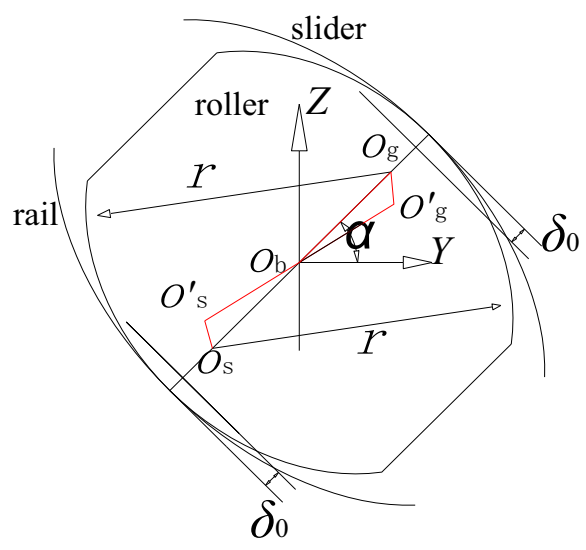

Figure.3 The geometry relationship of roller and rail

$$
O_{s} O_{g}=2 r-D_{w}=(2 f-1) D_{w}
$$

Where, $D_{w}$ is the diameter of the roller, $O_{\mathrm{s}}$ means the curvature center the slider rail. $O_{\mathrm{g}}$ means the curvature center the guide rail. $r=f D_{\mathrm{w}}$, and $f$ is the curvature ratio. $r$ is the curvature radius of roller paths in the slider and guide, Because of the elastic deformation of the sliding block and the guide rail is very small. The model will be considered as a rigid body, and only the deformation of the roller is considered. After the external loading, the curvature center coordinate of the $j$-th roller in the $j$-th column in the coordinate system of $O_{1}$ is $X_{i j(I)}, Y_{i j(l)}, Z_{i j(1)}$.

$$
\begin{aligned}
& X_{i j(1)}=W_{x}-\left(0.5 \cdot n \cdot D_{w}\right)+D_{w} \cdot j \\
& Y_{i j(1)}=\left\{\begin{array}{l}
W_{y}+L_{y}-0.5 \cdot(2 \cdot f-1) \cos \alpha, \quad i=1,2 \\
W_{y}-L_{y}+0.5 \cdot(2 \cdot f-1) \cos \alpha, \quad i=3,4
\end{array}\right. \\
& Z_{i j(1)}=\left\{\begin{array}{c}
L_{z}-0.5 \cdot(2 \cdot f-1) \sin \alpha, \quad i=1,4 \\
-L_{z}+0.5 \cdot(2 \cdot f-1) \sin \alpha, \quad i=2.3
\end{array}\right.
\end{aligned}
$$

Where, $n$ is the number of every column of guide. By the coordinate conversion, the coordinates obtained in the coordinate system can be expressed as equation (4):

$$
\left[X_{i j(0)} Y_{i j(0)} Z_{i j(0)} l\right]^{T}={ }^{0} T_{1}\left[X_{i j(l)} Y_{i j(l)} Z_{i j(l)} l\right]^{T}
$$

Also the curvature center coordinate of the $j$-th roller in the $i$-th column in the coordinate system of $O_{0}$ is $\mathrm{X}_{i j(0)}, Y_{i j(0)}, X_{i j(0)}$.

$$
\begin{aligned}
& X_{i j(\bar{\sigma})}^{\prime} X_{i \bar{j}} \varepsilon_{1} Y \quad{ }_{t} \varepsilon_{i} Z_{(1)} \quad y \\
& Y_{i j(\bar{\sigma})}^{\prime} \varepsilon X_{z} \quad i_{i j}^{+} Y_{1)}-\varepsilon_{i} Z_{(1)}+\delta_{x}
\end{aligned}
$$

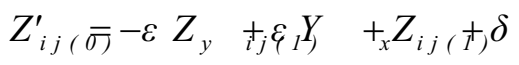

After receiving the loading, the distance between the curvature center of the slider and the 
curvature center of the guide is expressed below equation (6):

$$
O_{s}^{\prime} O_{g}^{\prime}=\left(Y_{i j(0)}-Y_{i j(0)}^{\prime}\right)^{2}+\left(Z_{i j(0)}-Z_{i j(0)}^{\prime}\right)^{2}
$$

The elastic deformation of each roller is equation (7):

$$
\delta_{i j}=\frac{O_{s}^{\prime} O_{g}^{\prime}-O_{s} O_{g}}{2}+\delta_{0}
$$

The model of contact force and roller elastic deformation base on hertz theory are showed below:

$$
\delta=K Q^{2 / 3}
$$

Where, $\mathrm{K}$ is parameters related to the curvature. The contact angle of the roller after deformation is:

$$
\alpha_{s i j}=\arctan \frac{\left|Z_{i j(0)}{ }^{\prime}-Z_{i j(0)}\right|}{\left|Y_{i j(0)}{ }^{\prime}-Y_{i j(0)}\right|}
$$

Therefore, the resultant forces of four columns rollers in slider 1 in $Y, Z$ axis directions are shown as equation (10):

$$
F_{l z}=\sum_{j=1}^{n}\left(Q_{1 j}+Q_{2 j}-Q_{3 j}-Q_{4 j}\right) \cdot \sin \alpha_{s i j} ; F_{1 y}=\sum_{j=1}^{n}\left(Q_{1 j}+Q_{2 j}-Q_{3 j}-Q_{4 j}\right) \cdot \cos \alpha_{s i j}
$$

In the same method of analysis, the resultant forces of all four columns rollers in slider 2, 3, 4 are obtained respectively in $Y$ axis, and $Z$ axis directions, which are represented as: $F_{2 y}, F_{3 y}, F_{4 y}, F_{2 z}, F_{3 z}$, $F_{4 z}$. Figure. 4 shows the situation of force and torque of the worktable installed four sliders. Force and torque analysis of the entire table lists the mechanical balance equations, as follow equation (11):

$$
\left\{\begin{array}{c}
F_{1 z}+F_{2 z}+F_{3 z}+F_{4 z}-F_{z}=0 \\
F_{1 y}+F_{2 y}+F_{3 y}+F_{4 y}-F_{y}=0 \\
W_{y}\left(F_{2 z}+F_{3 z}-F_{1 z}-F_{4 z}\right)+2 M_{x}+F_{z} d_{y}=0 \\
W_{x}\left(F_{1 z}+F_{2 z}-F_{3 z}-F_{4 z}\right)+2 M_{y}+F_{z} d_{x}=0 \\
W_{x}\left(F_{3 y}+F_{4 y}-F_{1 y}-F_{2 y}\right)+2 M_{z}+F_{\mathrm{y}} d_{x}=0
\end{array}\right.
$$
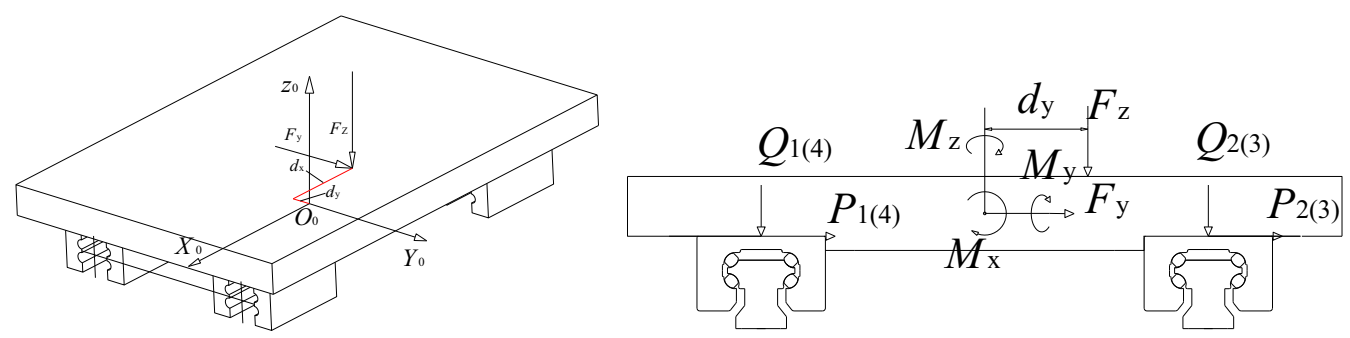

Figure.4 Stress analysis of linear feed unit 


\section{Calculating and Simulation}

By use computer, the vertical deformation and the lateral deformation of the linear feed unit are calculated. As shown In Figure.5, the thin line represent that it does not consider the change of contact angle. And the star line represent that it takes into the change of contact angle.

The tensional stiffness of the linear feed unit around $X_{0} 、 Y_{0}$ and $Z_{0}$ axis are calculated. As shown in Figure.6, the star line represent that it does not consider the change of contact angle. And the triangular line represent that it takes into the change of contact angle.
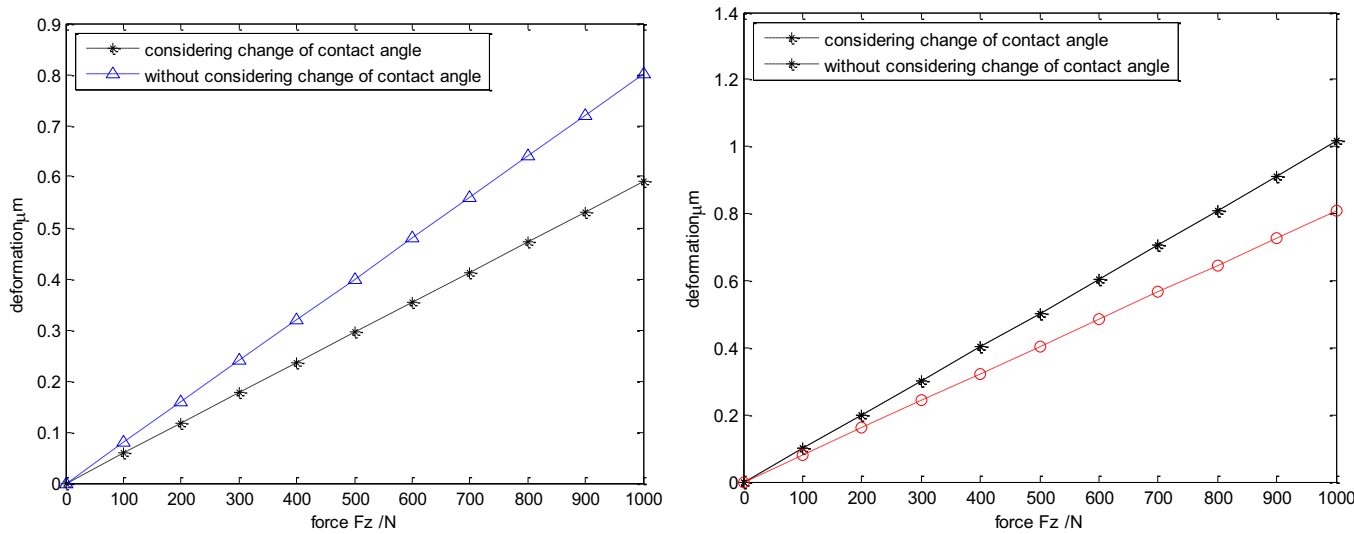

Figure.5 Deformation analysis of linear feed unit

The movement precision error of linear feed unit is showed as follow. In Figure.7, the curves show the three rotational errors respectively. The curves in Figure. 8 present two straightness errors and relationship between the value of the errors of three directions $X, Y, Z$ and the distant from force point to geometry center of feed unit.
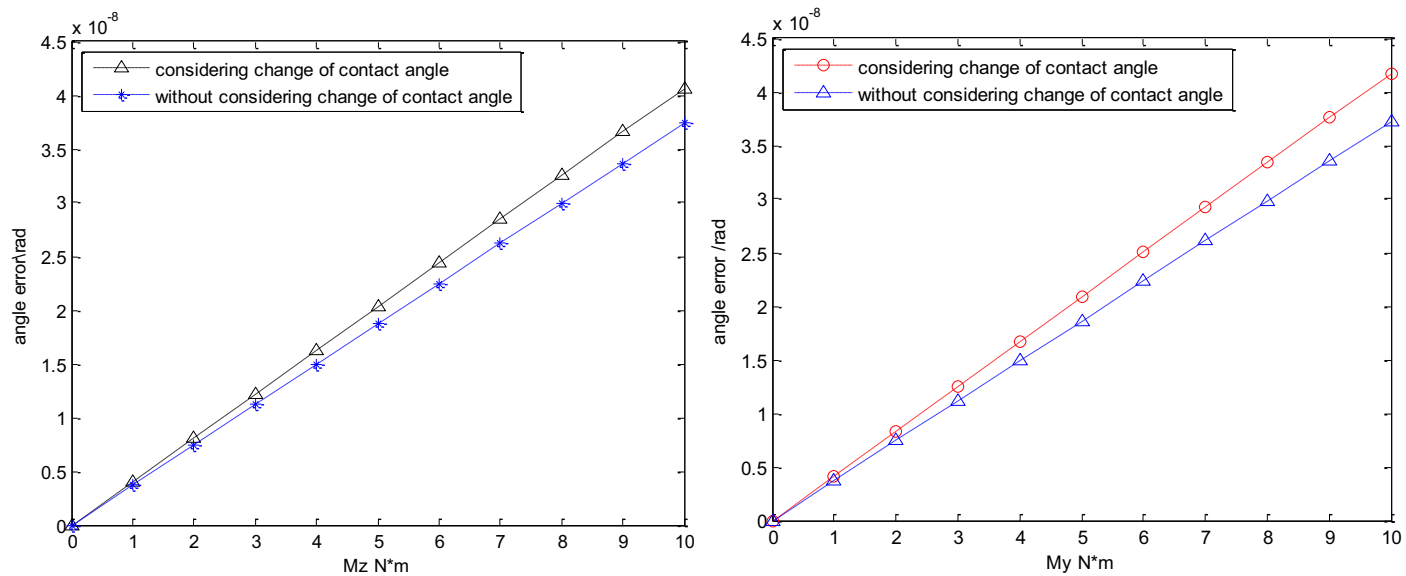

Figure.6 Deformation analysis of linear feed unit 

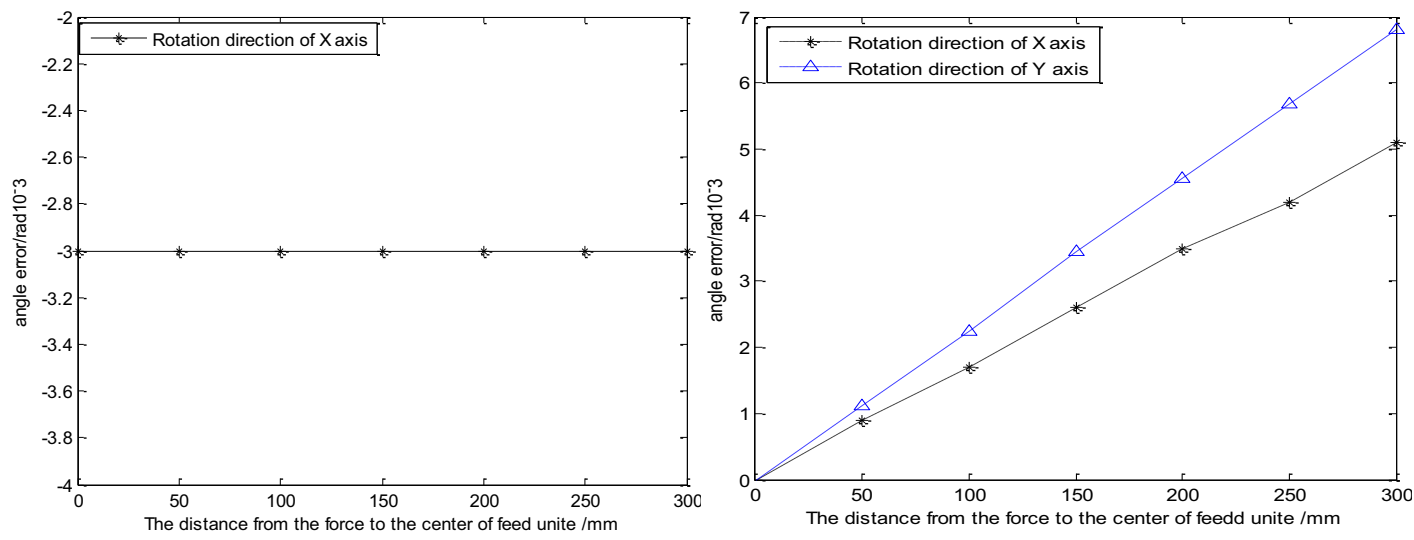

Figure.7 Angle errors and force acting point position
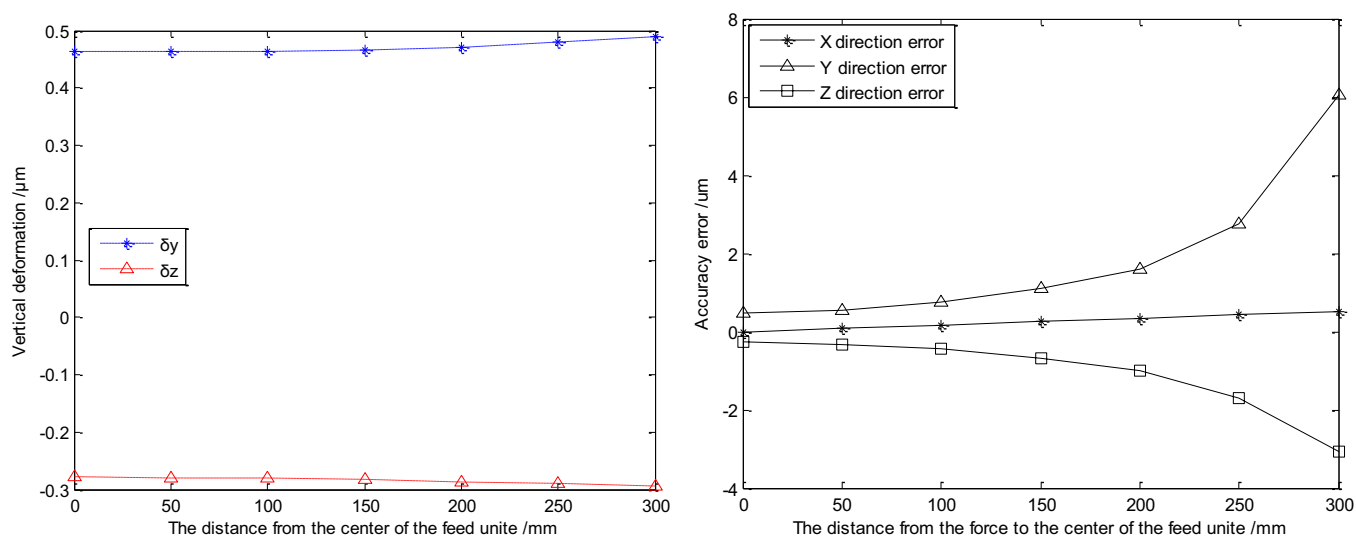

Figure.8 Errors and force acting point position

\section{Conclusions}

1. By comparing the analysis results, it shows that the vertical error and lateral error of the linear feed unit is greater than the error of in $X$ direction, because the movement of the linear feed unit is free in $X$ direction.

2. After considering the change of the contact angle, we found that, both vertical stiffness values and horizontal stiffness of linear feed unit are decrease. The larger the value of the contact angle change, the curve of the vertical stiffness and the curve of the horizontal stiffness is not consistent. the horizontal stiffness is falling even faster.

3. With the distant from force point to geometry center of feed unit become bigger, the angle error around $X, Y$, and $Z$ axis become bigger respectively. As shown in above section, the there angle error of the linear feed unit are very different. The angle error around $Y$ axis and the angle error around $Z$ axis are bigger the angle error around $X$ axis. The angle error around $Y$ axis is smaller the angle error 
around $Z$ axis.

4. When the load moves far from the geometry center of the linear feed unit, three rotational errors and two straightness accuracy errors all become bigger in different degrees.

\section{Acknowledgment}

This work is supported by National Natural Science Foundation of China, Grant No. 51575014; Natural Science Foundation of Beijing, Grant No. 3154029 and KZ201410005010; Important National Science \& Technology Specific Projects of China, Grant No. 2012ZX04010021-001-004; China Postdoctoral Science Foundation Funded Project, Grant No. 2016M591033; Beijing Postdoctoral Research Foundation, Grant No. 2015XX-13.

\section{References}

1. J.S.S. Wu, J.C. Chang, J.P. Hung, The effect of contact interface on dynamic characteristics of composite structures. J. Mathematics and Computers in Simulation 74 , 6 (2007), 454-467.

2. J.S.S. Wu, J.C. Chang, G.A. Tsai, C.Y. Lin, F.M. Ou, The effect of bending loads on the dynamic behaviors of a rolling guide. J. Journal of Mechanical Science and Technology 26 , 3 (2012), 671-680.

3. J.S. Liu, S.X. Xiong, Z. Li, J.L. Sun, The rigidity model of linear motion guide in the situation of LMGs standing under several loads. J. Proceedings of the International Conference on Mechanical Transmissions, (2001), pp. 664-666.

4. S. Shimizu, E. Saito, H. Uchida, Tribological studies of linear motion roller guide systems. J. Tribology Transactions 41, 1 (1998), 49-59.

5. S. Shimizu. Load distribution and accuracy/rigidity of linear motion roller guides system. J. Journal of the Japan Society for Precision Engineering 27, 2(1993), 119-125.

6. J. P Hung. Load effect on the vibration characteristics of a stage with rolling guides. J. Journal of Mechanical Science and Technology, 23, 1(2009), 9-99.

7. J.T Wei, Y Zhong. Model for wear prediction of roller guides. J. Wear (2013), 305:260-266 